\title{
Impact of Self-Heating Effect on Hot Carrier Degradation in High-Voltage LDMOS
}

\author{
Chih-Chang Cheng, J.F. Lin, Tahui Wang \\ Dept. of Electronics Engineering, National Chiao-Tung University, Hsin-Chu, Taiwan \\ T.H. Hsieh, J.T. Tzeng, Y.C. Jong, R.S. Liou, Samuel C. Pan, and S.L. Hsu \\ Taiwan Semiconductor Manufacturing Company, Science-Based Industrial Park, Hsin-Chu, Taiwan \\ E-mail: twang(a)cc.nctu.edu.tw
}

\begin{abstract}
Self-heating induced transient hot carrier effects in highvoltage n-LDMOS are investigated. A novel LDMOS structure incorporating a metal contact in the bird's beak region is fabricated, which allows us to probe an internal voltage transient in hot carrier stress. The AC stressfrequency dependence of device degradation is characterized and evaluated by a two-dimensional numerical simulation. Our result shows that drain current degradation in AC stress is more serious than in DC stress because of the reduction of self-heating effect.
\end{abstract}

\section{Introduction}

Laterally diffused MOS (LDMOS) transistors have been widely utilized in today's high-voltage/high-current drivers and RF power amplifiers [1,2]. Due to large power consumption, self-heating effect (SHE) is significant in a LDMOS. In this paper, we will study SHE on hot carrier degradation in $\mathrm{DC}$ and $\mathrm{AC}$ stress modes. To this purpose, we fabricate a special LDMOS structure, which incorporates a metal contact in the bird's beak region. Thus, we can probe an internal voltage $\left(\mathrm{V}_{\mathrm{I}}\right)$ transient due to SHE directly. Fig. 1 illustrates the device structure. Three regions of a LDMOS are indicated in the figure, including a channel region, an accumulation region and a field-oxide region. The contact is arranged in the accumulation region that the internal voltage $V_{I}$ can be used as a monitor for hot carrier effects in the channel. The contact area is small enough that the device electrical characteristics are not affected.

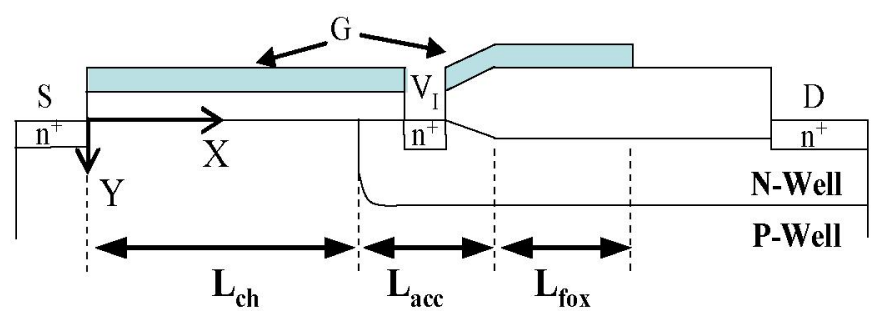

Fig. 1: Cross-section of a novel LDMOS structure. The metal contact $\left(V_{I}\right)$ is arranged in the accumulation region with $\mathrm{a}^{+}$implant. Three regions are indicated, including channel region $\left(\mathrm{L}_{\mathrm{ch}}\right)$, accumulation region $\left(\mathrm{L}_{\mathrm{acc}}\right)$, and field-oxide region $\left(\mathrm{L}_{\text {fox }}\right)$.
The device was processed in a $0.18 \mu \mathrm{m}$ CMOS technology with a gate oxide thickness of $100 \mathrm{~nm}$ and a channel length of $3 \mu \mathrm{m}$. The operation voltages are $\mathrm{V}_{\mathrm{g}}=40 \mathrm{~V}$ and $\mathrm{V}_{\mathrm{d}}=40 \mathrm{~V}$. To eliminate SHE in measurement, a fast transient measurement setup including a digital oscilloscope is built, as shown in Fig. 2. Linear drain current $\left(\mathrm{I}_{\mathrm{dlin}} @ \mathrm{~V}_{\mathrm{g}} / \mathrm{V}_{\mathrm{d}}=40 \mathrm{~V} / 0.1 \mathrm{~V}\right)$ is measured to monitor device degradation under $\mathrm{AC} / \mathrm{DC}$ stress. A threeregion charge pumping technique $[3,4]$ is used to locate hot carrier damage area in the device and to identify the type of generated oxide traps. Two-dimensional device simulation is performed to calculate a temperature distribution and corresponding hot carrier effects.

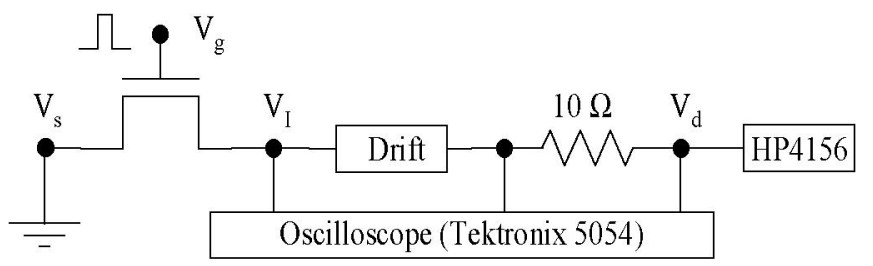

Fig. 2: Fast transient measurement setup for drain current and internal voltage $\left(\mathrm{V}_{\mathrm{I}}\right)$ characterization. The external resistance $(10 \Omega)$ is negligible compared to a total device resistance $(\sim 40 \mathrm{~V} / 10 \mathrm{~mA} \sim 4 \mathrm{k} \Omega)$. A gate pulse and constant $\mathrm{V}_{\mathrm{d}}$ are applied.

\section{Results and Discussion}

\section{(a) Self-Heating Characterization}

Fig. 3(a) shows a normalized drain current $\left(I_{d} / W\right)$ versus $\mathrm{V}_{\mathrm{d}}$ in small and large gate width devices in DC (Agilent 4156) measurement. The $I_{d} / W$ in a linear region is nearly the same, indicating no process variations in these two devices. However, the larger width device exhibits a smaller $I_{d} / W$ in the saturation region because of larger power consumption and thus a larger SHE. The reduction of the saturation current is attributed to self-heating induced mobility degradation $[5,6]$. Fig. 3(b) compares the $I_{d} / W$ from a DC and from a fast transient measurement for the large width device. A larger $\mathrm{I}_{\mathrm{d}} / \mathrm{W}$ is noticed in the transient measurement because of the elimination of SHE. In addition, SHE is manifested in the internal voltage measurement results by Agilent 4156 and by the fast transient setup (Fig. 4). The larger $\mathrm{V}_{\mathrm{I}}$ in a non-SHE 
condition is attributed to a higher mobility in accumulation region, thus resulting in a smaller drift region resistance. A larger internal voltage in non-SHE condition implies a stronger hot carrier stress in the channel region.
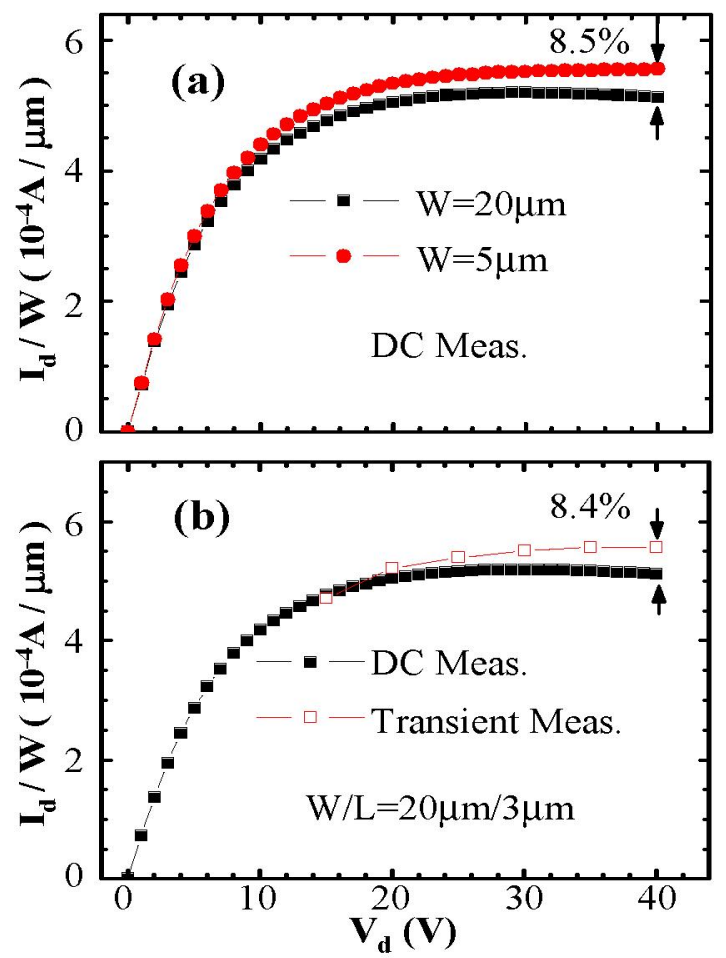

Fig. 3: (a) Normalized drain current $\left(I_{d} / W\right)$ versus drain voltage in small and large gate width devices in DC measurement (Agilent 4156). (b) The $I_{d} / W$ from a DC and a fast transient measurement for the large width device.

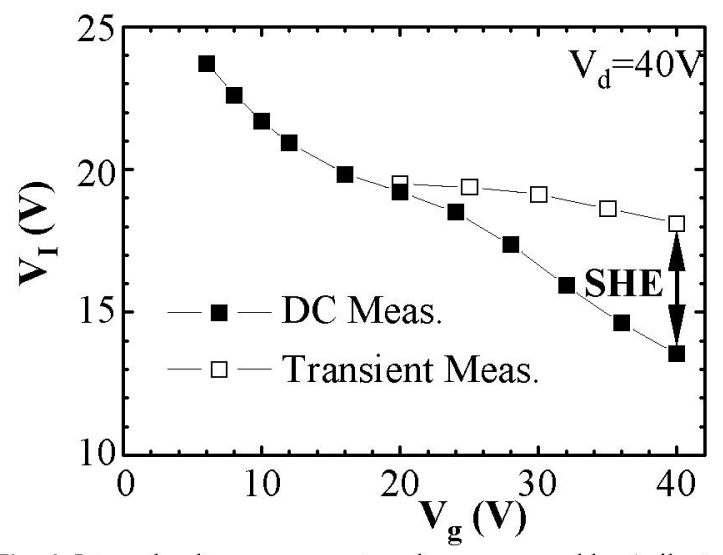

Fig. 4: Internal voltage versus gate voltage measured by Agilent 4156 and by a transient measurement setup.

\section{(b) Degradation Characteristics in AC/DC Stress}

Two stress modes (max. $I_{B}$, and max. $I_{g}$ ) are chosen in the study of hot carrier degradation in n-LDMOS. The $\mathrm{I}_{\mathrm{g}}-\mathrm{V}_{\mathrm{g}}$ and $I_{B}-V_{g}$ of a n-LDMOS are shown in Fig. 5. Fig. 6 shows $\mathrm{AC}$ and DC stress induced $\mathrm{I}_{\text {dlin }}$ degradations in the above two stress modes. Max. $\mathrm{I}_{\mathrm{B}}$ stress shows a slight difference in $\mathrm{I}_{\text {dlin }}$ degradation between $\mathrm{AC}$ and $\mathrm{DC}$ stresses, implying that SHE is not important at a lower stress $V_{\mathrm{g}}$. However, in maximum $\mathrm{I}_{\mathrm{g}}$ stress, $\mathrm{AC}$ stress shows much more $\mathrm{I}_{\text {dlin }}$ degradation than DC stress. Moreover, strong stress-frequency (Fig. 7(a)) and duty cycle (Fig. 7(b)) dependence is observed. In Fig. 7(a), the $I_{\text {dlin }}$ degradation increases with frequency and then becomes saturated. A corner frequency is found to be $f_{1}=20$ $\mathrm{kHZ}$ at a duty cycle $=10 \%$. In Fig. 8, we plot $\mathrm{I}_{\mathrm{dlin}}$ degradation versus pulse duration, i.e., duty cycle/frequency, in AC stress. A corner time of $\sim 5 \mu \mathrm{s}$ is obtained, suggesting that SHE becomes important as pulse duration is longer than $\sim 5 \mu \mathrm{s}$.

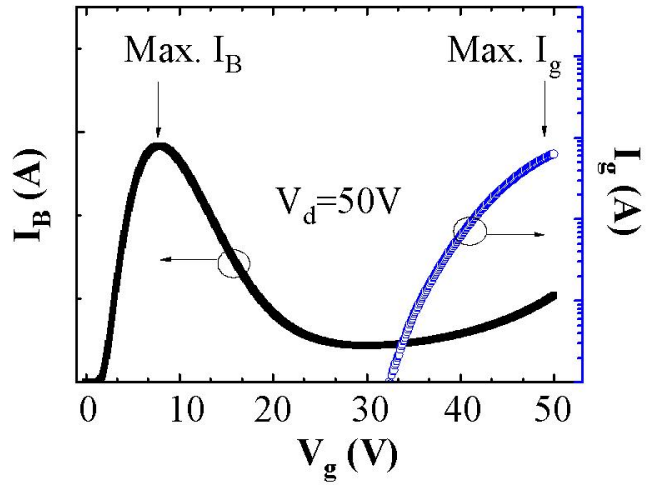

Fig. 5: Substrate current and gate current versus gate voltage in a LDMOS Two hot carrier stress modes are shown, maximum $\mathrm{I}_{\mathrm{B}}$ stress and maximum $\mathrm{I}_{\mathrm{g}}$ stress.

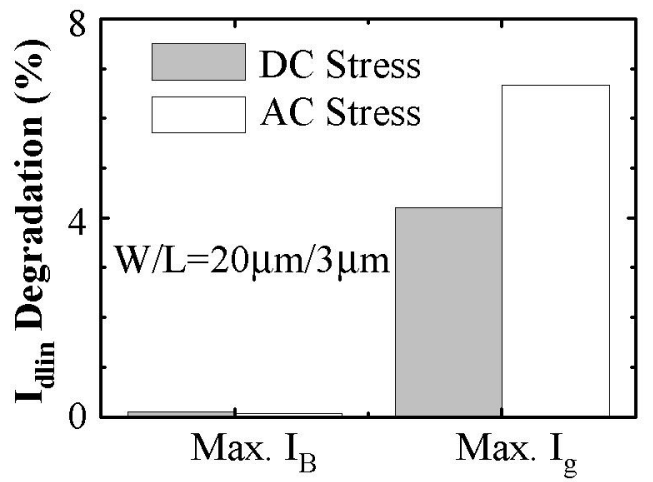

Fig. 6: Linear drain current degradation $\left(\mathrm{V}_{g} / \mathrm{V}_{\mathrm{d}}=40 \mathrm{~V} / 0.1 \mathrm{~V}\right)$ in two hot carrier stress modes. DC and $\mathrm{AC}$ stresses have the same cumulative stress time. AC stress has a frequency of $20 \mathrm{kHz}$ and a duty cycle of $10 \%$.

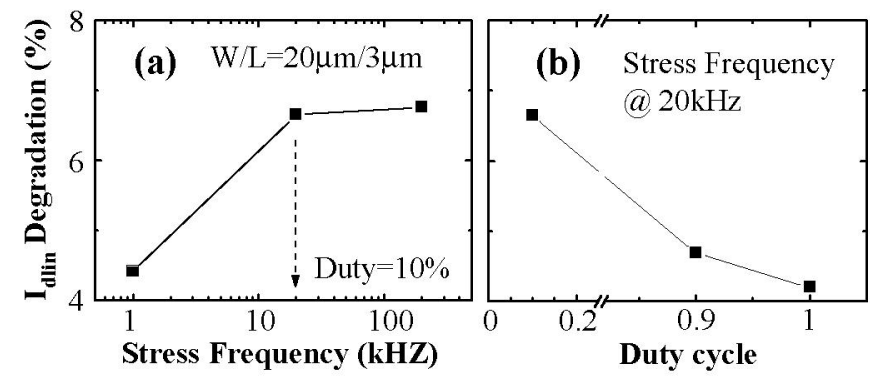

Fig. 7: (a) $\mathrm{I}_{\text {dlin }}$ degradation versus stress frequency with a duty cycle of $10 \%$. A corner frequency $\left(f_{l}\right)$ is around $20 \mathrm{kHz}$. (b) $\mathrm{I}_{\text {dlin }}$ degradation versus duty cycle for a frequency of $20 \mathrm{kHZ}$. 


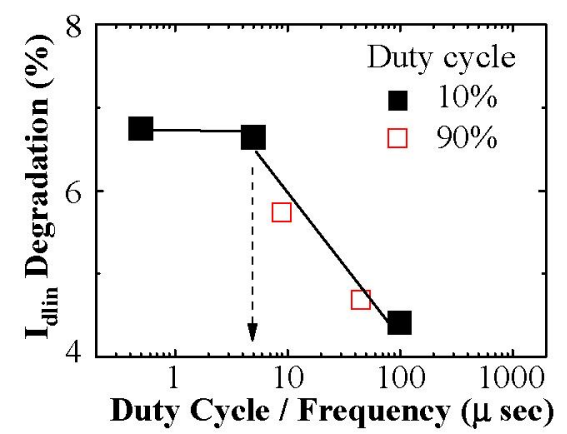

Fig. 8: Idlin degradation versus pulse duration (=duty cycle/frequency) in $\mathrm{AC}$ stress. The corner time is around $5 \mu \mathrm{s}$.

Fig. 9 shows a $\mathrm{V}_{\mathrm{I}}$ transient in a pulsed gate and $\mathrm{DC}$ drain voltage condition. The $\mathrm{V}_{\mathrm{I}}$ decreases with time due to SHE and the onset time of SHE is extracted to be around $5 \mu$ s. This result is consistent with the findings from $\mathrm{AC}$ stress induced degradation (Fig. 8).

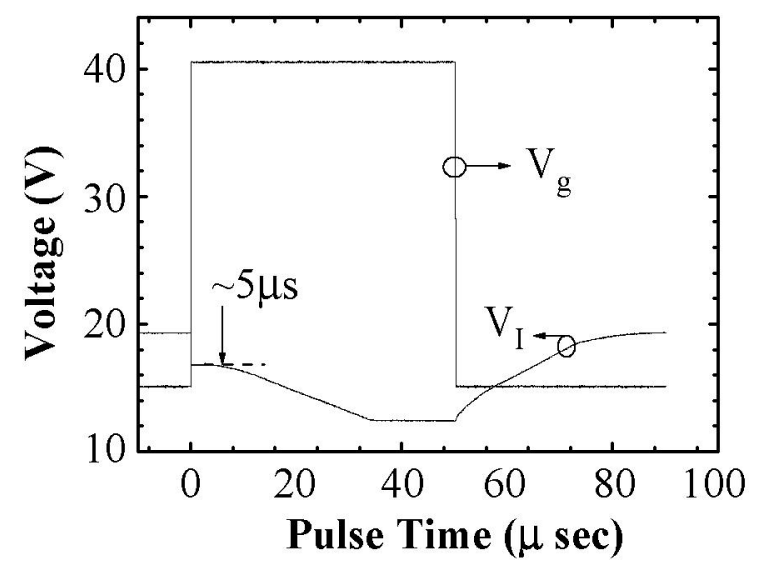

Fig. 9: The internal voltage $\left(V_{I}\right)$ transient in a pulsed gate and $D C$ drain voltage $\left(V_{d}=40 \mathrm{~V}\right)$ condition. The waveforms of $V_{I}$ and $V_{g}$ are plotted. The onset time for SHE is $\sim 5 \mu \mathrm{s}$.

A charge pumping measurement $\left(\mathrm{I}_{\mathrm{cp}}\right)$ result is shown in Fig. 10. A distinguished three-stage feature in $\mathrm{I}_{\mathrm{cp}}-\mathrm{V}_{\mathrm{gL}}$ is observed, corresponding to the three regions of a LDMOS respectively. By comparing the pre-stress and post-stress $\mathrm{I}_{\mathrm{cp}}$ in each stage, we are able to separate interface trap $\left(\mathrm{N}_{\mathrm{it}}\right)$ and fixed oxide charge $\left(Q_{o x}\right)$ creation in each region of the device. The result in Fig. 10 reveals that $\mathrm{AC}$ stress generates more $\mathrm{N}_{\mathrm{it}}$ in the channel region and more $\mathrm{Q}_{\mathrm{ox}}$ in the accumulation region. The larger trap generation rate in $\mathrm{AC}$ stress results from a smaller temperature rise and thus a larger stress gate current (Fig. 11). The self-heating induced temperature change in a LDMOS is simulated by a two-dimensional numerical device simulation (Fig. 12). The ambient temperature in the simulation is $300 \mathrm{~K}$. In Fig. 12, the drift region shows a higher temperature change than channel region, implying a larger mobility degradation and thus a higher drift region resistance. The SHE is stronger at a higher $\mathrm{V}_{\mathrm{g}}$. The simulation also reveals a higher $\mathrm{V}_{\mathrm{I}}$ in a non-SHE condition (Fig. 13). Good agreement between measurement and simulation is obtained in Fig. 13. Because of a larger $V_{\text {I }}$ in the non-SHE condition, the simulated impact ionization rate is stronger in the non-SHE condition (Fig. 14). This feature also confirms our charge-pumping results in Fig. 10 and concludes a more serious $\mathrm{I}_{\mathrm{dlin}}$ degradation rate in $\mathrm{AC}$ stress (Fig. 15).

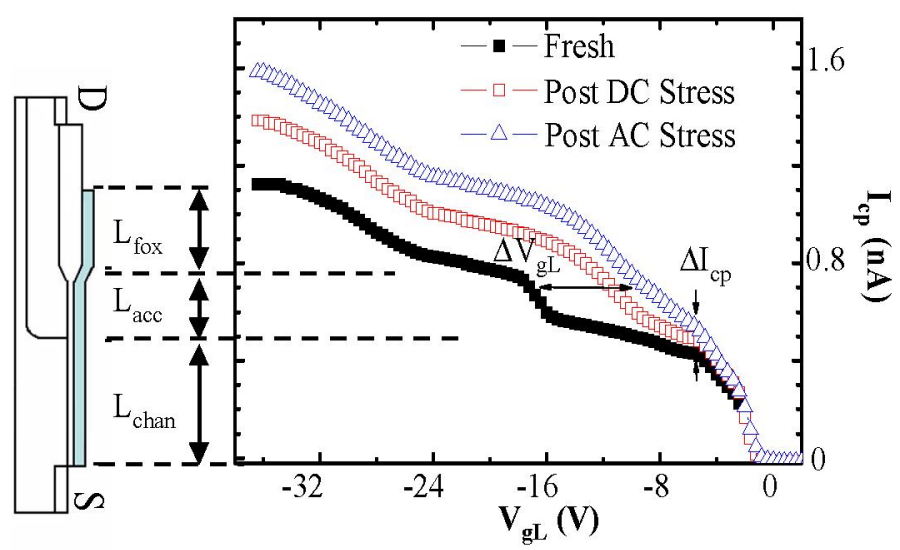

Fig. 10: Three-region charge pumping measurement results after maximum $I_{g} A C$ and DC stress. A $V_{\text {gL }}$ shift is in the accumulation region and a $I_{c p}$ increase is in the channel region. For more details, see $[3,4]$.

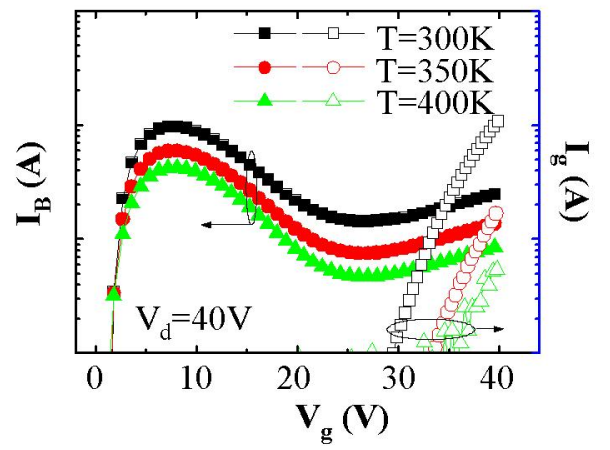

Fig. 11: Substrate current and gate current versus gate voltage for different temperatures.

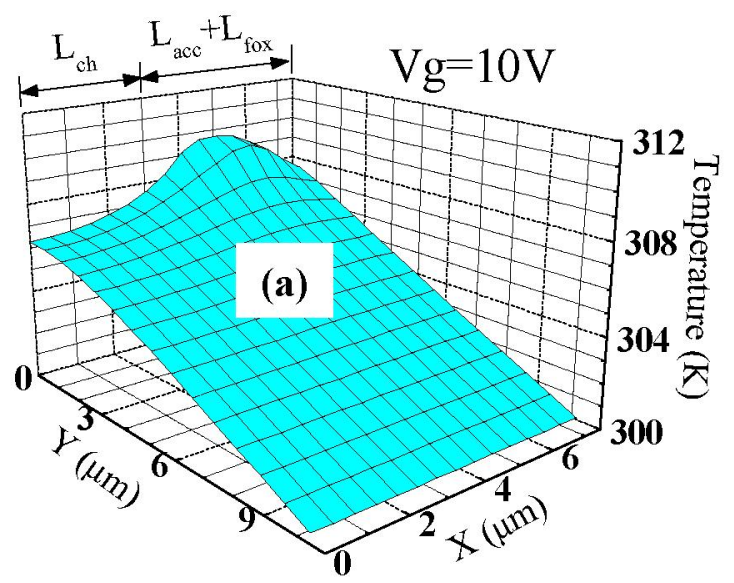




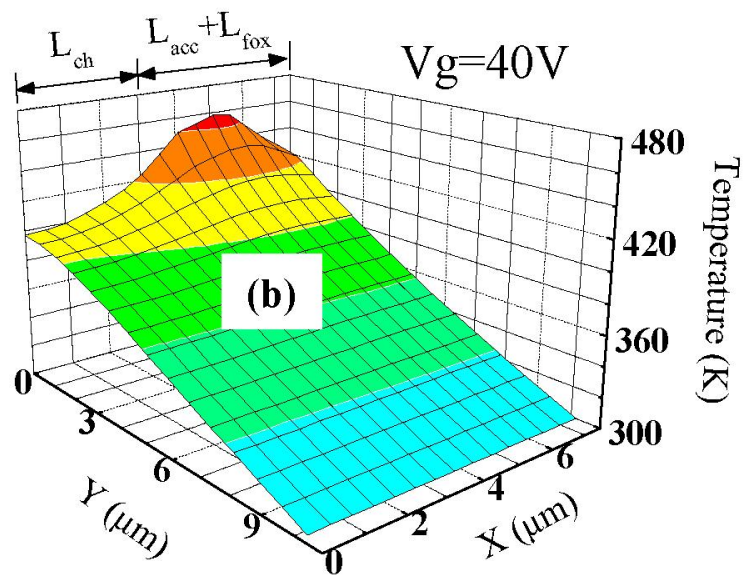

Fig. 12: Simulation of a temperature distribution with SHE. The ambient temperature is $300 \mathrm{~K}$. $\mathrm{X}$ and $\mathrm{Y}$ axes are indicated in Fig. 1. (a) $\mathrm{V}_{\mathrm{g}} / \mathrm{V}_{\mathrm{d}}=10 \mathrm{~V} / 40 \mathrm{~V}$. (b) $\mathrm{V}_{\mathrm{g}} / \mathrm{V}_{\mathrm{d}}=40 \mathrm{~V} / 40 \mathrm{~V}$.

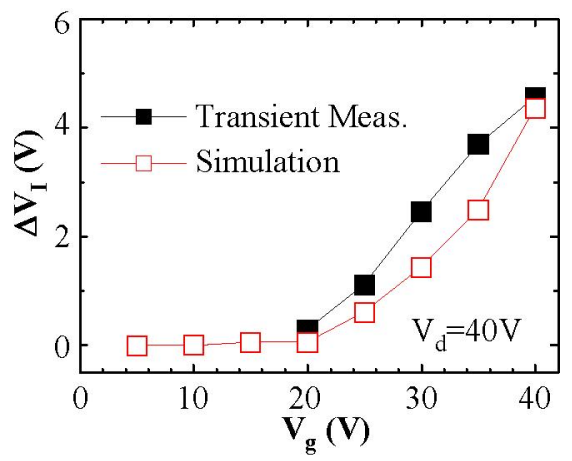

Fig. 13: Internal voltage change $\left(\mathrm{V}_{\mathrm{I}}\left(\right.\right.$ non-SHE)- $\left.\mathrm{V}_{\mathrm{I}}(\mathrm{SHE})\right)$ versus gate voltage from measurement and from simulation.
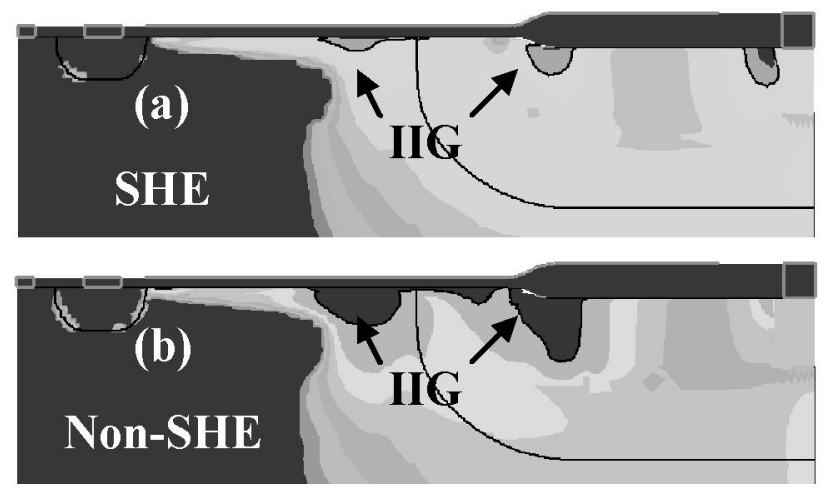

Fig. 14: Two-dimensional device simulation of impact ionization generation (IIG) rate at $\mathrm{V}_{\mathrm{g}} / \mathrm{V}_{\mathrm{d}}=40 \mathrm{~V} / 40 \mathrm{~V}$. (a) SHE is included and (b) SHE is not included.

\section{Conclusion}

Transient self-heating effect in AC hot carrier stress in LDMOS has been studied by measuring an internal voltage. The extracted self-heating time is around $5 \mu \mathrm{s}$. The AC stress at maximum $I_{g}$ yields the worst hot carrier degradation because of the elimination of self-heating effect.

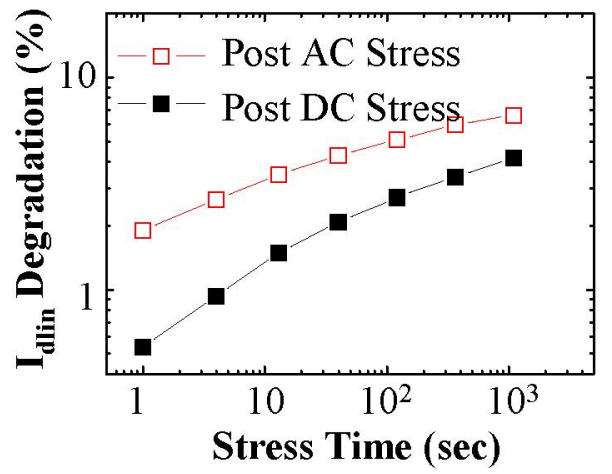

Fig. 15: $I_{d l i n}$ degradation rate after $A C$ and $D C$ stress in maximum $I_{g}$ stress condition. The AC stress frequency is $20 \mathrm{kHz}$ and the duty cycle is $10 \%$.

\section{Acknowledgments}

The authors of NCTU would like to acknowledge financial and technical support from NSC under contract \# NSC 95-2221-E-009-303 and TSMC JDP. The authors also would like acknowledge National Center for HighPerformance Computing for providing the device simulation environment.

\section{References}

[1] D. Muller, A. Giry, F. Judong, C. Rossato, F. Blanchet, B. Szelag, A. Monroy Aguirre, R. Sommet,D. Pache, and O. Noblanc, "HighPerformance 15-V Novel LDMOS Transistor Architecture in a $0.25-\mu \mathrm{m}$ BiCMOS Process for RF-Power Applications," IEEE Trans. on Electron Devices, vol. 54, pp.861-868, April, 2007.

[2] Jongdae Kim, Tae Moon Roh, Sang-Gi Kim, Q. Sang Song, Dae Woo Lee, Jin-Gun Koo, Kyoung-Ik Cho, and Dong Sung Ma, "High-Voltage Power Integrated Circuit Technology Using SOI for Driving Plasma Display Panels," IEEE Trans. on Electron Devices, vol. 48, pp.12561263, June, 2001.

[3] Chih-Chang Cheng, K.C. Tu, Tahui Wang, T.H. Hsieh, J.T. Tzeng, Y.C. Jong, R.S. Liou, Sam C. Pan, and S.L. Hsu, "Investigation of Hot Carrier Degradation Modes in LDMOS by Using a Novel Three-Region Charge Pumping Technique," IEEE International Reliability and Physics Symposium (IRPS), pp.334-337, 2006.

[4] Chih-Chang Cheng, J. F. Lin, Tahui Wang, T.H. Hsieh, J.T. Tzeng, Y.C. Jong, R.S. Liou, Samuel C. Pan, and S.L. Hsu "Physics and Characterization of Various Hot Carrier Degradation Modes in LDMOS by Using a Three-Region Charge Pumping Technique," IEEE Trans. on Device and Materials Reliability, pp.358-363, 2006.

[5] Emil Arnold, Howard Pein, and Sam P. Herko, "Comparison of SelfHeating Effects In Bulk-Silicon and SO1 High-Voltage Devices," IEEE International Electron Device Meeting (IEDM), pp.813-816, 1994.

[6] Gary M. Dolny, Gerald E. Nostrand, and Kevin E. Hill, "The Effect of Temperature on Lateral DMOS Transistors in a Power IC Technology," IEEE Trans. on Electron Devices, vol. 39, pp.990-995, April, 1992. 